\title{
Tips for young cardiologists or aspiring cardiologists
}

\section{Oscar M.P. Jolobe}

Manchester, United Kingdom

Address for correspondence:

Dr Oscar M P Jolobe

Flat 6 Souchay Court

I Clothorn Road

Manchester

M20 6BR

United Kingdom

Email:

oscarjolobe@yahoo.co.uk

\section{INTRODUCTION}

The popular perception is that it takes heroic surgery (including interventional cardiology) to save someone's life, and that other branches of the profession, including those engaged in primary care, have nothing heroic to offer. But the starting point of any heroic enterprise must be diagnosis, including diagnosis at primary care level. The following examples and references to the literature illustrate the crucial importance of attention to the detail of eliciting ordinary physical signs that lead to correct diagnosis. That attention to detail should be an enduring component of the medical curriculum.

Examples in my personal experience and in the medical literature:

To begin with, I am reminded of a patient who consulted me when I was a general practitioner at Mthatha between 1967 and 1973. He had recent onset breathlessness, pyrexia, finger clubbing, and the murmur of aortic regurgitation. After the necessary urgent phone calls had been made, the patient was airlifted by helicopter to Wentworth Hospital (Durban) for the aortic valve replacement that saved his life. In that context, the starting point was the routine inspection of the hands for finger clubbing, and the identification of the murmur of aortic regurgitation which, in some circumstances, can be the most elusive murmur in cardiology. Ironically, it is in the life-threatening context that the murmur may behave atypically, thereby compounding the difficulty of reaching the correct diagnosis of its underlying cause. The 2 life-threatening underlying causes of an atypical murmur of aortic regurgitation are aortic leaflet rupture in infective endocarditis, and aortic regurgitation resulting from aortic dissection. In both circumstances aortic regurgitation is of acute onset. Acute-onset regurgitation generates a haemodynamic state where the early diastolic murmur becomes softer and more difficult to detect. Diagnostic difficulty is compounded by the fact that the heart sounds, themselves, become softer, and it becomes more difficult to distinguish diastole from systole.(1) Even in this age of high-tech diagnostics, it is the frontline doctors in primary care, and in the emergency department (we call it Accident and Emergency Department) who need to have a high index of suspicion for acute-onset aortic regurgitation. That index of suspicion comes from years of disciplined auscultation for heart murmurs. That discipline is now being eroded by colleagues who are prepared to conduct a clinical examination (including auscultation for heart murmurs) without taking the precaution to ask the patient to take his shirt off.(2) I experienced this at first hand when I attended a private clinic after falling ill on one of my visits to South Africa. The stethoscope was simply placed over my shirt.

The other worthwhile routine is the correct evaluation of jugular venous pressure. Again, referring to my Mthatha days as a general practitioner, I recall a young man who came in complaining of abdominal distension and bilateral lower limb oedema. Nevertheless, he was able to lie down on the couch without becoming breathless. On examination, his jugular venous pressure was elevated up to the level of his ear lobes even when he was sitting bolt upright. I dispatched him off to Wentworth again, with a diagnosis of constrictive pericarditis, the latter an eminently reversible cause of congestive heart failure if diagnosed and treated early. The "give away" sign of constrictive pericarditis was that this patient conformed to the axiom which states that "the presence of distended neck veins in a patient who is able to lie comfortably in the recumbent posture is a characteristic feature of the disease"(3) namely, constrictive pericarditis. Another axiom is that, in constrictive pericarditis, the jugular venous pressure remains persistently (I would even say "stubbornly") elevated (and markedly so) even when peripheral oedema has been substantially reduced by diuretic therapy. ${ }^{(4)}$ That is the physical sign that primary care doctors must look out for when they are treating heart failure with diuretics. Pulmonologists must also be on the lookout for 
this eminently treatable disease. Chronic "idiopathic" pleural effusion responds amazingly well to the simple expedient of identification of the physical sign where "the peaks of the jugular venous pulse (JVP) are higher than the angle of the mandible even when the patient is sitting up."(5) The latter report was yet another example of constrictive pericarditis presenting under the guise of pleural effusion. ${ }^{(6)}$ Hepatologists must also take note. Patients who have been referred to the hepatology clinic for investigation of ascites are often able to lie comfortably on the examination couch without getting out of breath. However, in some of these patients, it is only on sitting bolt upright that the jugular venous pulse can be seen "tickling" the patient's ear lobes, a physical sign that should prompt urgent referral to a cardiologist. ${ }^{(7)}$

In our account of the catalogue of life-saving physical signs, we must not forget the peripheral pulses. The appropriate anecdote that comes to mind is that of the lady whose daughter phoned me one day in the middle of my ward round. She said her mother had, that very morning, experienced sudden onset numbness in her left foot. I knew her mother well. She had atrial fibrillation and had undergone mitral valve replacement in the past. She was now on oral anticoagulants. Fortunately, I also knew her pedal pulses well, having documented them in her clinical notes in the past. On examination, this time, the left foot had no palpable pedal pulses. The consequence was that the lady was promptly dispatched to the vascular surgeons for embolectomy, which saved her leg. I still have a landscape painting by her daughter hanging in my living room, which she gave me as a token of gratitude for saving her mother's leg. The takeaway lesson here is that prior knowledge of the state of the peripheral pulses is a great asset to correct diagnosis. Furthermore, notwithstanding the classical description of embolic limb ischaemia as being characterised, not only by pulselessness, but also by severe pain, pallor, paralysis, and coolness to touch, ${ }^{(8)}$ embolic peripheral limb ischaemia can be a totally painless affair. ${ }^{(9)}$ The converse is the clinical presentation of mesenteric embolism, in which severe abdominal pain is the cardinal symptom despite initially total absence of objective clinical signs such as abdominal rigidity and rebound tenderness. I encountered that scenario in one of my posts as a junior doctor, when I had a patient with atrial fibrillation who was admitted with a history of sudden onset severe abdominal pain. Surprisingly, the abdomen was soft, there was no rebound tenderness, and bowel sounds were present. This "disconnect" between the severity of abdominal pain (with the patient writhing in agony) and the paucity of a comparable degree of severity of objective physical signs is the hallmark of the initial stages of mesenteric embolism. ${ }^{(10)}$ It is at that stage that the bowel can still be saved.
Abdominal distension, rebound tenderness, and absence of bowel sounds will come later, when neither the bowel nor the patient's life can be saved.

The humble blood pressure is the final stop in my catalogue of routine physical signs which might one day save someone's life. In 1998 Kaplan wrote: "The measurement of blood pressure is likely the clinical procedure of greatest importance that is performed in the sloppiest manner." (II) What was true in 1998 is still true to this day. Nurses, and not doctors, should be measuring blood pressure because they can take their time to do it properly. ${ }^{(12)}$ Each medical practice and each outpatient clinic should have a dedicated nurse practitioner who has been specially trained in the correct technique ${ }^{(13)}$ of measurement of blood pressure, and that nurse should be the one who measures the blood pressure in each patient who passes through that clinic. Even when the SPRINT methodology ${ }^{(13)}$ for blood pressure measurement must be adapted to local conditions, the bare minimum should be measurement of blood pressure after 5 minutes rest in a quiet room, followed by 3 measurements 60 seconds apart. The correct blood pressure will be the average of those three measurements. Furthermore, in every emergency department, each patient who comes in with chest pain (including chest pain with ST segment elevation) should have the blood pressure measured in both arms so as to improve the detection rate for aortic dissection, ${ }^{(14)}$ including aortic dissection characterised by ST segment elevation.(15) In a patient with chest pain the association of marked interarm blood pressure difference and aortic regurgitation should ring "alarm bells" for aortic dissection. In turn, those alarm bells should trigger urgent point of care transthoracic echocardiography, transoesophageal echocardiography or computed chest tomography angiography, depending on availability or logistics. Each hospital should compile its own algorithm and flow chart for such an eventuality

\section{CONCLUSION}

At all levels of expertise physical signs matter hugely. Both in primary care and in secondary and tertiary care doctors should constantly be fine tuning their technique of eliciting physical signs. The subtle ones, such as the murmur of aortic regurgitation, are the ones which specially need constant practice. You can never have too many "goes" at eliciting that murmur. The moment you tire of listening to that murmur is the moment you embark on the downhill course which takes you to the occasions where you might miss the opportunity to save a life.

\section{Conflict of interest: none declared.}




\section{REFERENCES}

I. Hamirani Y, Dietl CA, Voyles W, et al. Acute aortic regurgitation. Circulation 2012; I26: I| |2|-| | 26.

2. Van Galen LS, Bos PPP. You can keep your shirt on. European Journal of Internal Medicine 2018;52:E39-E40.

3. Evans W, Jackson F. Constrictive pericarditis. British Heart Journal 1952; 14:53-69.

4. Conti CR, Friesinger GC. Chronic constrictive pericarditis. Clinical and laboratory findings in I I cases. Johns Hopkins Med J 1967; I 20:262-274.

5. Akhter MW, Nono IN, Rahimtoola SH. Constrictive pericarditis masquerading as chronic idiopathic pleural effusion: Importance of physical examination. Am J Med 2006; 1 19:el-e4.

6. Jolobe OMP. Disproportinonate elevation of jugular venous pressure in pleural effusion. British Journal of Hospital Medicine 201 1;72:582-585.

7. Reed MC, Dhaliwal G, Saint S, et al. The right angle. N Engl J Med 20। I 364:1350- 1356.

8. Braun R, Lin M. Acute limb ischaemia: A case report and literature review. Journal of Emergency Medicine 2015;49:1011-1017.

9. Jolobe OMP. Acute leg ischaemia may present without pain in some cases. BMJ 20 I3;DOI: I0.1 1 36/bmj.f3930.

10. Bala M, Kashuk J, Moore EE, et al. Acute mesenteric ischaemia guideline of the World Society of Emergency Surgery. World Journal of Emergency Surgery 2017;12:Article number 38(2017). https://doi.org/|0.1 |86/s | 3017. 017-0150-5.

II. Kaplan NM. Commentary on the sixth report of the Joint National Committee (JNC 6). AM J Hypertens 1998; 1 1:134- 36.

12. Jolobe OMP. Mythmaking in the measurement of blood pressure European Journal of Internal Medicine 20 I 4;25:el I.

13. Myers MG, Clouthier L, Gelfer M, et al. Blood pressure measurement in the post-SPRINT era. A Canadian experience. Hypertension 20 | 6;68:25।-253.

14. Patel PD, Arora RR. Pathophysiology, diagnosis, and management of aortic dissection. Therapeutic advances in cardiovascular disease 2008;21:439-468

15. Wang J-L, Chen C-C, Wang C-Y, et al. Acute type A aortic dissection presenting as ST-segment elevation myocardial infarction referred for primary percutaneous coronary intervention. Acta Cardiol Sin 2016;32:265-272. 